\title{
The immune-modulatory effects of exercise should be favorably harnessed against COVID-19
}

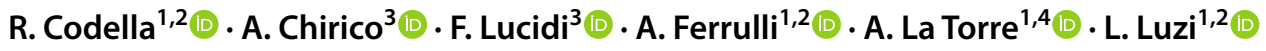

Received: 7 July 2020 / Accepted: 22 August 2020 / Published online: 3 September 2020

(C) Italian Society of Endocrinology (SIE) 2020

Keywords Physical activity $\cdot$ Metabolism $\cdot$ Immune modulation $\cdot$ Exercise immunology

$\begin{array}{ll}\text { Abbreviations } \\ \text { AMPK } & \text { 5' AMP-activated protein kinase } \\ \text { GLP-1 } & \text { Glucagon-like peptide 1 } \\ \text { IGF } & \text { Insulin-like growth factor } \\ \text { IL } & \text { Interleukin } \\ \text { NK } & \text { Natural killer } \\ \text { NF-אB } & \text { Nuclear transcription factor } \\ \text { TNF } & \text { Tumor necrosis factor } \\ \text { URTI } & \text { Upper respiratory tracts infections }\end{array}$

What COVID-19 lockdowns mean to the possibility of being physically active

This COVID-19 crisis imposes a revolution of habits and lifestyles that present-day societies appear unprepared to. Social distancing may be naturally associated with reduced levels of physical activity: home residing may augment sedentary behaviors (sitting time, watching-tv, using smartdevices). In turn, this may result in the worsening of chronic diseases such as obesity, type 2 diabetes, hypertension and the deleterious effects of aging. These syndromic features are all pooled within a dysmetabolic continuum for which regular physical activity of moderate intensity is salutary. In other words, low physical fitness, dysmetabolic conditions (in primis, obesity), and an altered immune system could be

R. Codella

roberto.codella@unimi.it

1 Department of Biomedical Sciences for Health, Università degli Studi di Milano, Via Fratelli Cervi 93, Segrate (Milano), 20090 Milan, Italy

2 Department of Endocrinology, Nutrition and Metabolic Diseases, IRCCS MultiMedica, Milan, Italy

3 Department of Social and Developmental Psychology, Sapienza University of Rome, Rome, Italy

4 IRCCSIstituto Ortopedico Galeazzi, Milan, Italy seriously adverse for a subject exposed to this coronavirus, SARS-CoV-2.

Moreover, consequences induced by societal changes in response to such a pandemic viral infection may aggravate psychosocial issues by weakening immunological defenses ("psychoneuroimmunity") [1].

\section{The harmful effects of physical inactivity}

Countless studies have documented how abnormal responses from the immune system and the triggering of local inflammation, even at low-grade, in key cells and tissues, are the primum movens for the development of chronic metabolic diseases [2]. Obesity, strictly linked to sedentary behaviors, is an evident example of this. In addition, the involvement of cell types from both adaptive and immune immunity in the visceral adipose tissues of obese humans and mice underpins an immune-metabolic milieu, for which obesity-related insulin resistance and inflammation may have a proper autoimmune component [3]. The "disuse syndrome" encompasses low mechanical load, energy expenditure and metabolic rate, therefore, implying metabolic derangements, ranging from insulin resistance and inflammation to impairments in insulin signaling, adipose tissue lipolysis and mitochondrial functions [4]. Acutely, a concomitant increase of sedentary behaviour and physical inactivity impairs multi-organ insulin sensitivity and cardiorespiratory fitness - reversiblyand may be accompanied by concurrent dyslipidaemic profiles with fat accumulation at liver and central sites [5]. Even reducing the daily step count and step intensity has been associated with all-cause mortality and may lead to metabolic disorders in healthy adults [6].

However, during crisis like COVID-19, human physiology is not the sole one to be rapidly impaired by physical inactivity. Physical inactivity per se deteriorates mood profiles and has been associated with poor mental wellness. 
Although a clear psychological impact of COVID-19 is fairly unknown, either infected patients or asymptomatic individuals may experience anxiety, depression, stigma, anger, frustration [7]. These psychological variables may reduce immunity and negatively affect recovery.

\section{The favorable immune-metabolic modulation induced by exercise}

Regular exercise and/or physical activity have been proved to elicit a wide range of health-promoting effects, bearing preventive, therapeutic, and sometimes even reverting actions, in a multitude of diseases.

Exercise induces a cascade of events, mostly favorable, levering the immune system, via an ultimately anti-inflammatory pattern within organ crosstalk (Fig. 1).

Skeletal muscle-Although IL-6 is itself a proinflammatory cytokine, it leads to an anti-inflammatory balance. In contracting muscles, marked increases of myokine IL-6 determine a downregulation of proinflammatory TNF as well as an upregulation of anti-inflammatory cytokines interleukin (IL)-10 and IL-1 receptor antagonist (IL-1ra). Moreover, IL-6, being pleiotropic, exerts systemic effects by enhancing insulin secretion via GLP-1; insulin-stimulated glucose uptake (through AMPK activation) both in skeletal muscle and adipose tissue; lipid oxidation peripherally and whole-body. Circulating levels of IL-15 are also increased in skeletal muscle following acute resistance training and enact protective responses by modulating cells of both the innate (NK) and adaptive immune systems (T cells).

Brain-Exercise augments endogenous $\beta$-endorphin levels in cerebrospinal fluid, increasing natural killer (NK)cell-mediated cytotoxicity in vivo. Through the hypothalamic-pituitary-adrenal axis activation, adrenal cortex and medulla are stimulated to release cortisol and adrenaline, respectively. These two hormones inhibit proinflammatory TNF cytokines by monocytes.

Heart and lungs-Adaptations to exercise training improve cardiorespiratory fitness, spanning from hemodynamic to hormonal changes. These result in improved oxygen capacity (uptake and delivery), larger breathing reserve and greater respiratory muscle efficiency. Myocardial function assessed at maximum minute volume is increased with training as well as systemic arterial compliance and stroke volume. Blood pressure reduction is brought by diminishing vasoconstriction and catecholamine levels. In fact, the sympathetic nervous and the renin-angiotensin systems are calmed by training. Muscle cytochrome C oxidase activity is also boosted by exercise leading to reduce local expression of proinflammatory cytokines and inducible nitric oxide synthase while augmenting insulin-like growth factor (IGF-1).

Gut-A growing body of evidence suggests that moderate increments of physical fitness ensure integrity of the intestinal barrier permeability by short-chain fatty acids activation, and an overall anti-inflammatory profile even in chronic and immune-based diseases.

Multiple organs are implicated in a crosstalk fashion during exercise, initiating diverse anti-inflammatory responses.

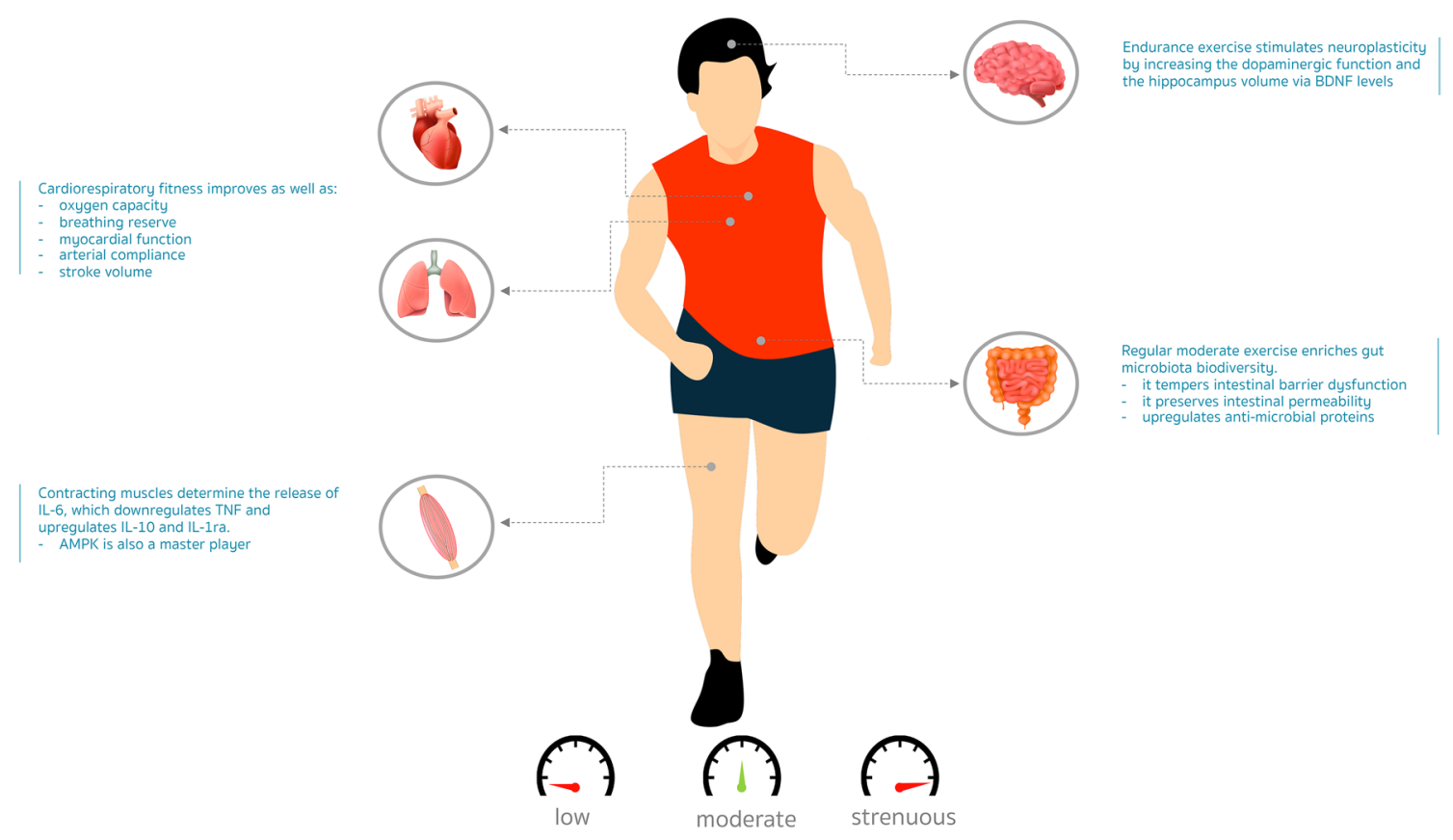

Fig. 1 Exercise-induced organ crosstalk. Beneficial effects are maximized at doses corresponding to moderate intensity, regular frequency 


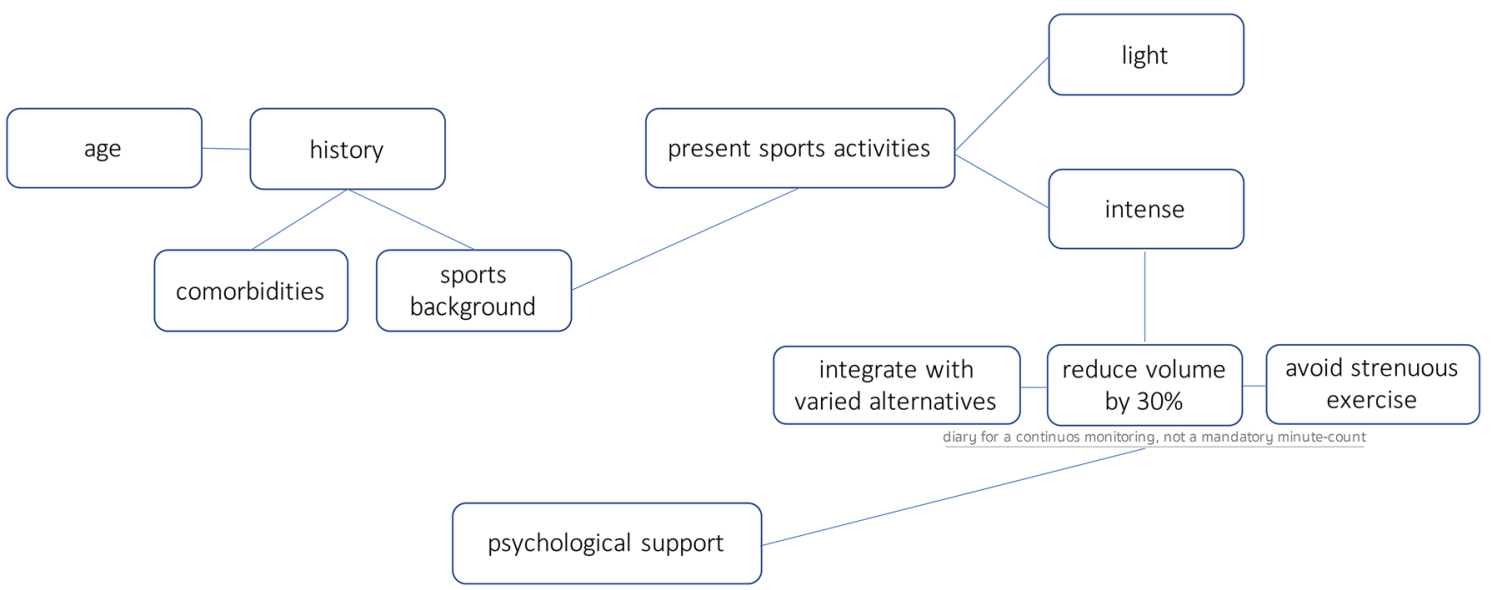

Fig. 2 Diagram of the anamnestic triage for a personalized exercise prescription during pandemics, such as COVID-19 outbreak

\section{Physical activity at the crossroad between prevention and vulnerability}

An inverse relationship does exist between volume of physical activity and all-cause mortality rates in men, women, younger and older adults. However, highest volumes of strenuous exercise may become concerning from an immunological standpoint.

It has been suggested that the cytokine response to strenuous exercise can be viewed as a model of the cytokine response to a traumatic event like sepsis or an extraordinarily intense "fight-or-fight" situation. Early report on COVID-19 described an associated upsurge of proinflammatory cytokines, including IL-1 $\beta$ and IL-6 [8]. In addition to IL-6, other inflammatory biomarkers, such as C-reactive protein, serum ferritin and coagulation index, and D-dimer were found elevated in patients with COVID-19 and diabetes compared with those without diabetes, indicating that patients with comorbidities are more prone to an inflammatory storm with a possible degeneration of COVID-19 [9]. Alike, absolute counts of lymphocytes, erythrocytes, and hemoglobin were all diminished among patients with COVID-19 and diabetes compared to those without diabetes [9]. These stressors resemble a post-traumatic status, so does the cytokine response of an extreme or ultra-prolonged exercise (e.g. marathon running or Ironman triathlon) that could be associated with muscle damage and even systemic endotoxemia.

Beyond the "open-window" period, in which the upper respiratory tracts infections (URTI) risk is greater, hyperventilation of a typical running of strenuous intensity may exacerbate the pattern of breathing, with a potential inhalation of several agents, including the SARS-CoV-2 to deepest areas of the lungs. If infection occurs, the exercise-increased air flow could hypothetically expedite the virus diffusion to the lower airways and alveoli during the incubation period [10].
The line of demarcation between immunoprotection and immunodepression is thin, thus an exercise strategy must be cautiously orchestrated, considering the adjunct risk represented by immunosenescence.

One lesson that can be learnt by this pandemic is that not only responses to diseases are heterogenous but also responses to exercise may vary across populations depending on a multitude of factors. Either the physical fitness status, the characteristics of the training, or the individual immunological integrity and responsiveness may be harshly predicted, controlled and finally managed. For these reasons, now more than ever, a targeted combination of competences and expertise are highly warranted, encompassing a teamwork of physicians, psychologists and exercise scientists.

Therefore, an anamnestic triage for a personalized exercise prescription during a SARS-CoV-2 outbreak is envisaged (Fig. 2).

\section{Healthcare policies to harness the pleiotropic actions of exercise during pandemics}

Certainly, government health directives have become more unified in their response to this coronavirus. Countries enabled coordinated actions, in terms of capacities regarding both health and social support.

Whilst many excellent digital resources and online exercise recommendations have been readily available, more in-depth exercise prescriptions should be progressively developed as far as they now are being largely applied to diversified societal, sports and clinical contexts.

According to our representative sample of 2400 web-surveyed people, aged 18-67, the prevalence of physical inactivity in Italy has moved from $8 \%$, before the outbreak, to $23 \%$ after this virus surge (interviews administered through 
17-22 March 2020) [11]. In the quarantine period, modalities of training and workouts were radically changed, performed with no equipment (44\%), tutored by social network (13\%) and video calling (9\%), or followed by on-purpose apps (11\%) [11].

During lockdowns, people are home confined. Although home-based exercises are safe and may vary extensively, performing physical activity outdoor has been shown to amplify the beneficial effects of exercise [12]. Besides, lack of commuting time should be virtuously replaced, because, at present, like in quarantine periods, it is worryingly converted in physical inactivity. An ultimate set of factors pertains the mandatory graduality of the exercise schedules, which should be substantially diminished as to intensities and volumes (Fig. 2), according to the individual sports background: long-lasting periods of detraining, comparable to periods of reduced responsiveness (e.g. aging, spaceflight, injuries) may result in impaired performance and/or augmented injury risk.

It is important to understand the underlying mechanisms behind the positive effects of exercise, whether is moderate or intense, tailored on individualized immunological responses (which are upstream mediated by preexisting physiological and psychological conditions). The fascinating challenge will be whether we can capture the intimate mechanisms by which exercise comes to the forefront in the battle against pandemics like COVID-19.

Acknowledgements This work was supported by Italian Ministry of Health Ricerca Corrente-IRCCS MultiMedica.

Author contributions All authors were responsible for drafting the manuscript and revising it critically for valuable intellectual content. All authors approved the version to be published.

\section{Compliance with ethical standards}

Conflict of interest The authors declare that they have no competing interests.

Ethics approval This is a comment article. Its original data are referenced in Chirico et al. [11] and contained in a public repository [https://osf.io/wscmr/]. The questionnaire and methodology for those data were approved by the Ethical Committee of University "La Sapienza", Department of Psychology of Development and Socialization Processes.

Informed consent All participants provided informed consent prior to their participation.

\section{References}

1. Kim S-W, Su K-P (2020) Using psychoneuroimmunity against COVID-19. Brain Behav Immun. https://doi.org/10.1016/j. bbi.2020.03.025

2. Hotamisligil GS (2006) Inflammation and metabolic disorders. Nature 444:860-867. https://doi.org/10.1038/nature05485

3. Mathis D, Shoelson SE (2011) Immunometabolism: an emerging frontier. Nat Rev Immunol 11:81-83. https://doi.org/10.1038/ nri2922

4. Alibegovic AC, Sonne MP, Højbjerre L et al (2010) Insulin resistance induced by physical inactivity is associated with multiple transcriptional changes in skeletal muscle in young men. Am J Physiol Metab 299:E752-E763. https://doi.org/10.1152/ajpen do.00590.2009

5. Bowden Davies KA, Sprung VS, Norman JA et al (2018) Shortterm decreased physical activity with increased sedentary behaviour causes metabolic derangements and altered body composition: effects in individuals with and without a first-degree relative with type 2 diabetes. Diabetologia 61:1282-1294. https://doi. org/10.1007/s00125-018-4603-5

6. Saint-Maurice PF, Troiano RP, Bassett DR et al (2020) Association of daily step count and step intensity with mortality among US adults. JAMA 323:1151. https://doi.org/10.1001/ jama.2020.1382

7. Brooks SK, Webster RK, Smith LE et al (2020) The psychological impact of quarantine and how to reduce it: rapid review of the evidence. Lancet 395:912-920. https://doi.org/10.1016/S0140 $-6736(20) 30460-8$

8. Conti P, Ronconi G, Caraffa A et al (2020) Induction of proinflammatory cytokines (IL-1 and IL-6) and lung inflammation by Coronavirus-19 (COVI-19 or SARS-CoV-2): anti-inflammatory strategies. J Biol Regul Homeost Agents. https://doi.org/10.23812 /CONTI-E

9. Guo W, Li M, Dong Y et al (2020) Diabetes is a risk factor for the progression and prognosis of COVID-19. Diabetes Metab Res Rev. https://doi.org/10.1002/dmrr.3319

10. Matricardi PM, Negro RWD, Nisini R (2020) The first, comprehensive immunological model of COVID-19: implications for prevention, diagnosis, and public health measures. (preprint)

11. Chirico A, Lucidi F, Galli F et al (2020) COVID-19 outbreak and physical activity in the Italian population: a cross-sectional analysis of the underlying psychosocial mechanisms. Front Psychol. https://doi.org/10.3389/fpsyg.2020.02100

12. Manferdelli G, la Torre A, Codella R (2019) Outdoor physical activity bears multiple benefits to health and society. J Sports Med Phys Fitness. https://doi.org/10.23736/S0022-4707.18.08771-6

Publisher's Note Springer Nature remains neutral with regard to jurisdictional claims in published maps and institutional affiliations. 